\title{
Consumo de álcool em binge por adolescentes escolares de 12 anos de idade e sua associação com sexo, condição socioeconômica e consumo de álcool por melhores amigos e familiares
}

\author{
Binge drinking among 12-year-old adolescent schoolchildren \\ and its association with sex, socioeconomic factors \\ and alcohol consumption by best friends and family members
}

Paula Cristina Pelli Paiva ${ }^{1}$

Haroldo Neves de Paiva ${ }^{1}$

Joel Alves Lamounier ${ }^{2}$

Efigênia Ferreira e Ferreira ${ }^{3}$

Carlos Augusto Santos César ${ }^{2}$

Patrícia Maria Zarzar ${ }^{4}$

${ }^{1}$ Departamento de

Odontologia, Universidade

Federal dos Vales do

Jequitinhonha e Mucuri

(UFVJM). Rua da Glória

187 Campus I. 38610-000

Diamantina MG Brasil

paulacpp@ig.com.br

${ }^{2}$ Programa de Pós-

Graduação em Ciências

da Saúde, Faculdade de

Medicina, Universidade

Federal de Minas Gerais

(UFMG).

${ }^{3}$ Departamento de Saúde

Coletiva, Faculdade de

Odontologia, UFMG.

${ }^{4}$ Departamento de

Odontopediatria e

Ortodontia, Faculdade de

Odontologia, UFMG.
Abstract This is a cross-sectional study with a convenience sample of 101 twelve-year-old adolescents enrolled in public and private schools in the city of Diamantina in the State of Minas Gerais. The scope was to evaluate the prevalence of binge drinking among 12-year-old schoolchildren and its association with gender, socioeconomic status and alcohol consumption by family members and best friends. The participants completed a self-administered questionnaire entitled the Alcohol Use Disorders Identification Test (AUDIT-C) and the consumption of alcoholic beverages by friends and family. Parents/guardians answered the form on sociodemographic questions. Descriptive analyses and association tests were performed ( $p<0.05)$. The prevalence of binge drinking was $24.8 \%$. Alcoholic beverage consumption began at the age of 10 (16.8\%), though sex was not associated with binge drinking by adolescents. However, attending a public school (0.005) and alcohol consumption by best friends $(p<000.1)$ were associated with binge drinking by adolescents in the bivariate analysis. The prevalence of binge drinking was high and was associated with low socioeconomic status and alcohol consumption by the best friend. No association between sex and alcohol consumption by the family members of adolescents was detected.

Key words Alcoholic beverages, Binge drinking, Adolescent, Prevalence
Resumo Estudo transversal com amostra de conveniência de 101 adolescentes com 12 anos, de escolas públicas e privadas de Diamantina/MG, avaliando a prevalência de consumo de álcool em "binge" por adolescentes escolares de 12 anos e sua associação com as condições socioeconômicas, sexo, consumo de bebidas alcoólicas por familiares e melhores amigos. Os participantes responderam questionário autoaplicável, Teste de Identificação de Problemas Relacionados ao Uso de Álcool C (Audit-C) e sobre consumo de álcool por amigos e familiares. O formulário sobre questões sociodemográficas foi respondido pelos pais/responsáveis. Foram feitas análises descritivas e teste de associação $(p<0,05)$. A prevalência do consumo em "binge" foi de 24,8\%. O início do consumo de bebida alcoólica foi aos 10 anos (16,8\%). O sexo não esteve associado ao consumo de álcool pelos adolescentes. Entretanto, estudar em escola pública $(0,005)$ e consumo de álcool pelo melhor amigo ( $p=0,0001)$ estiveram associados ao consumo em "binge", por adolescentes na análise bivariada. $O$ consumo em "binge" foi alto e associado à condição socioeconômica baixa e ao consumo de álcool pelo melhor amigo. Não foi observada associação com o sexo e o consumo de álcool pelos familiares dos adolescentes.

Palavras-chave Bebidas alcoólicas, Bebedeira, Adolescente, Prevalência 


\section{Introdução}

O consumo de bebida alcoólica entre adolescentes constitui um importante problema social e de saúde pública, pois apresenta alta prevalência e início cada vez mais precoce, sendo preocupação crescente em muitos países' ${ }^{1}$. A adolescência, considerada pela Organização Mundial de Saúde como o periodo de 10-19 anos, engloba tanto as modificações biológicas corporais como, também, as transformações psicossociais ${ }^{2}$. É uma fase marcada por grandes descobertas e instabilidade emocional, período crítico para o desenvolvimento de competências pessoais e interpessoais, aquisição de habilidades para atuar e tomar decisões, consubstanciando a personalidade ${ }^{3}$. Devido à fase de desenvolvimento, o adolescente pode ser particularmente suscetível a influências sociais, destacando-se a importância da família, da escola e dos grupos de pares e, dependendo do contexto social, pode adotar comportamentos de proteção ou risco para a saúde $e^{4,5}$.

O consumo de bebida alcólica em "binge" é definido como a ingestão de cinco doses ou mais em uma única ocasião ${ }^{6}$ e vem se tornando um padrão comum de consumo de álcool entre os adolescentes. Principalmente pelo período de vulnerabilidade em que vivem, nesta faixa etária, na qual as relações interpessoais estão se firman$\mathrm{do}^{7}$, este tipo de comportamento pode ser considerado por si só como de alto risco.

As bebidas alcoólicas são as drogas psicotrópicas mais utilizadas entre os adolescentes, embora legalmente seu consumo seja permitido somente após os 18 anos (Lei no 9.294, de 15 de junho de 1996) $)^{8}$.

Uma série de fatores pode influenciar o comportamento de beber em "binge" entre os adolescentes, tais como a necessidade de socialização, os relacionamentos com o sexo oposto, expectativas e crenças e, acima de tudo, contextos familiares e sociais $^{9-14}$.

O consumo de bebida alcoólica reduz o autocontrole e aumenta o risco ${ }^{15}$ para o comportamento antissocial, o crime, o mau desempenho escolar, a violência interpessoal e as lesões acidentais. O consumo em "binge" é a principal causa de lesões (incluindo as resultantes de acidentes de trânsito), violência (violência doméstica e interpessoal) e mortes prematuras ${ }^{16}$. As características de consumo de bebidas alcoólicas em "binge" associado ao nível socioeconômico, foram descritas em um estudo realizado nas capitais brasileiras com adolescentes (14-18 anos). A prevalência do consumo em "binge" foi $32 \%$ no último ano e o maior risco de envolvimento em bebedeira foi relatado para os estudantes do sexo masculino, pertencentes à classe social alta, mais velhos e que frequentavam escolas privadas ${ }^{17}$. A prevalência de consumo em "binge" e sua associação com diferentes redes de amigos, sexo e nível socioeconômico foi investigada em amostra de 891 adolescentes (15-19 anos), em Belo Horizonte. O consumo em "binge" foi relatado por $36 \%$ da amostra, sendo que $26,2 \%$ reportaram adotar este padrão de consumo, entre menos que uma vez por mês a mensalmente e $9,9 \%$ semanalmente à diariamente. Além disto, este comportamento foi associado ao sexo masculino, baixa vulnerabilidade socioeconômica e tipo de rede de amigos $^{11}$.

Fatores como a baixa condição socioeconômica e a presença de laços familiares onde há ausência de comunicação, suporte e disciplina estão associados ao consumo de álcool em "binge" por adolescentes, constituindo-se como fatores de risco para o consumo abusivo de bebidas alcoólicas ${ }^{12-14,18}$. Não há consenso na literatura em relação à condição socioeconômica e ao consumo em "binge". Estudos que exploraram estas variáveis apresentaram resultados conflitantes, necessitando que novos estudos sejam desenvolvidos para esclarecer melhor esta questão.

Estudos revelam a associação deste consumo em "binge" por adolescentes com envolvimento em brigas, em eventos violentos e acidentes automobilísticos, podendo resultar desde traumatismos corporais e dentários até, em alguns casos extremos, em morte ${ }^{12,13}$. A importância de estudos abordando uma faixa etária mais nova, período inicial da adolescência, pode orientar melhor a implantação de medidas de prevenção e controle do consumo em "binge" pelos adolescentes para a prevenção de suas consequências.

Baseado na precocidade com que os adolescentes estão iniciando o consumo de bebidas alcoólicas e a escassez de estudos realizados nesta idade, a presente pesquisa avaliou a prevalência de consumo em "binge" por adolescentes de 12 anos de idade e sua associação com condição socioeconômica, sexo, bem como, o consumo de bebidas alcoólicas por familiares e amigos.

\section{Método}

O presente estudo transversal foi realizado em Diamantina, município com aproximadamente 46.372 habitantes, localizado ao norte do estado de Minas Gerais, sudeste do Brasil. O município 
possui taxa de alfabetização de $83,4 \%$ e índice de desenvolvimento humano (IDH) de 0,716 , considerado pelo programa das nações unidas para o desenvolvimento (Pnud), como sendo o melhor índice dentre as cidades pertencentes ao Vale do Jequitinhonha (IBGE).

O estudo foi aprovado pelo Comitê de Ética e Pesquisa da Universidade Federal de Minas Gerais. As autorizações das escolas participantes foram obtidas, bem como o termo de consentimento livre e esclarecido assinado pelos participantes e seus pais ou responsáveis. Os participantes tiveram sua confidencialidade garantida.

A coleta de dados aconteceu nos meses de fevereiro e março de 2013. A amostra de conveniência foi constituída por todos os alunos com 12 anos completos, matriculados em 02 escolas públicas e 01 particular da zona urbana da cidade, totalizando 101 escolares. As escolas foram selecionadas por conveniência, levando em consideração a localização geográfica, com o objetivo de incluir escolares de classes socioeconômicas distintas.

Questionários para coleta das variáveis socioeconômicas foram enviados aos pais ou responsáveis, juntamente com o termo de consentimento livre e esclarecido. O presente estudo adotou a renda familiar investigada pelo número de salários mínimos recebidos por todos os membros economicamente ativos residentes com o adolescente, categorizado pela mediana, bem como a escolaridade da mãe.

Por se tratar de um estudo direcionado a escolares de 12 anos de idade, foi aplicado a versão curta do instrumento Audit (Teste para Identificação de Problemas Relacionados ao Uso de Álcool), o teste Audit-C, composto por perguntas relacionadas à frequência e à quantidade do consumo de álcool, com respostas pontuadas de 0 a $12^{19}$. O questionário Audit- $\mathrm{C}$ foi validado no Brasil $^{20}$, sendo composto das seguintes perguntas: 1. Com que frequência você consomiu bebidas alcoólicas no último ano?; 2. Quantas doses de álcool você consome num dia normal? e 3. Com que frequência você consome cinco ou mais doses em uma única ocasião? As opções de respostas para a primeira questão, segundo o instrumento foram: Nunca, uma vez por mês ou menos, 2-4 vezes por mês, 2-3 vezes por semana, 4 ou mais vezes por semana. Para a questão 2: 1, 2 ou 3, 4 ou 5, 6 ou 7, 8 ou mais e para a questão 3: Nunca, Menos que uma vez por mês, Uma vez por mês, Uma vez por semana, Diariamente ou quase todos os dias. $\mathrm{O}$ instrumento foi aplicado em sala de aula, na ausência do professor.
O consumo em "binge" foi definido como o consumo de 5 doses ou mais em uma única ocasião ${ }^{6}$, com as respostas dicotomizadas em: 0 para quem nunca consumiu em "binge" e 1 para quem consumiu de uma vez por mês ou menos até diariamente ou quase todos os dias. Também foi questionado aos escolares a frequência de consumo de álcool por amigos e familiares, bem como a idade que experimentou bebidas alcoólicas pela primeira vez ${ }^{17}$. A percepção do adolescente sobre o controle dos pais também foi investigada, com as perguntas: Meu pai controla tudo o que faço? Minha mãe controla tudo o que faço? Para a resposta utilizou-se escala de Likert, com as opões de concordo, nem concordou nem discordo e discordo ${ }^{21}$.

Para a obtenção dos resultados foram realizadas análises descritivas dos dados, para caracterização da amostra e em seguida análise bivariada adotando o teste do Qui-quadrado, com nível de significância $\mathrm{p}<0,05$, através do programa Statistical Package for Social Sciences 19.0 (SPSS).

\section{Resultados}

A amostra de conveniência foi de 101 escolares, provenientes de uma escola particular e duas públicas. A Tabela 1 apresenta as características demográficas e socioeconômicas.

A prevalência de consumo de álcool na vida e consumo em "binge" foi de $37,6 \%$ e $24,8 \%$ respectivamente. A média de idade do primeiro consumo de álcool foi de 10,75 anos.

O sexo e o controle dos pais não estiveram associados ao consumo de álcool pelos escolares (Tabela 2). Entretanto, a condição socioeconômica, medida pela renda familiar e escolaridade da mãe, esteve associada estatísticamente com o consumo em "binge", sendo que os escolares da classe social baixa, que estudavam em escolas públicas, com mãe que só cursou até o ensino fundamental, tiveram mais chance de pertencerem ao grupo que consumiram bebidas alcoólicas em "binge" (Tabela 2).

A frequência de consumo de álcool pelo melhor amigo $(p=0,0001)$ esteve associada ao consumo em "binge" por adolescentes na análise bivariada (Tabela 3 ). O consumo de bebida alcoólica pelo pai $(\mathrm{p}=0,262)$, mãe $(\mathrm{p}=0,292)$ e irmão $(p=0,056)$ não estiveram associados ao consumo em "binge" pelo escolar (Tabela 3). Adolescentes cujos melhores amigos consumiram bebidas alcoólicas apresentaram 10,49 vezes mais chance (95\%IC 3,41-32,22) de pertencerem 


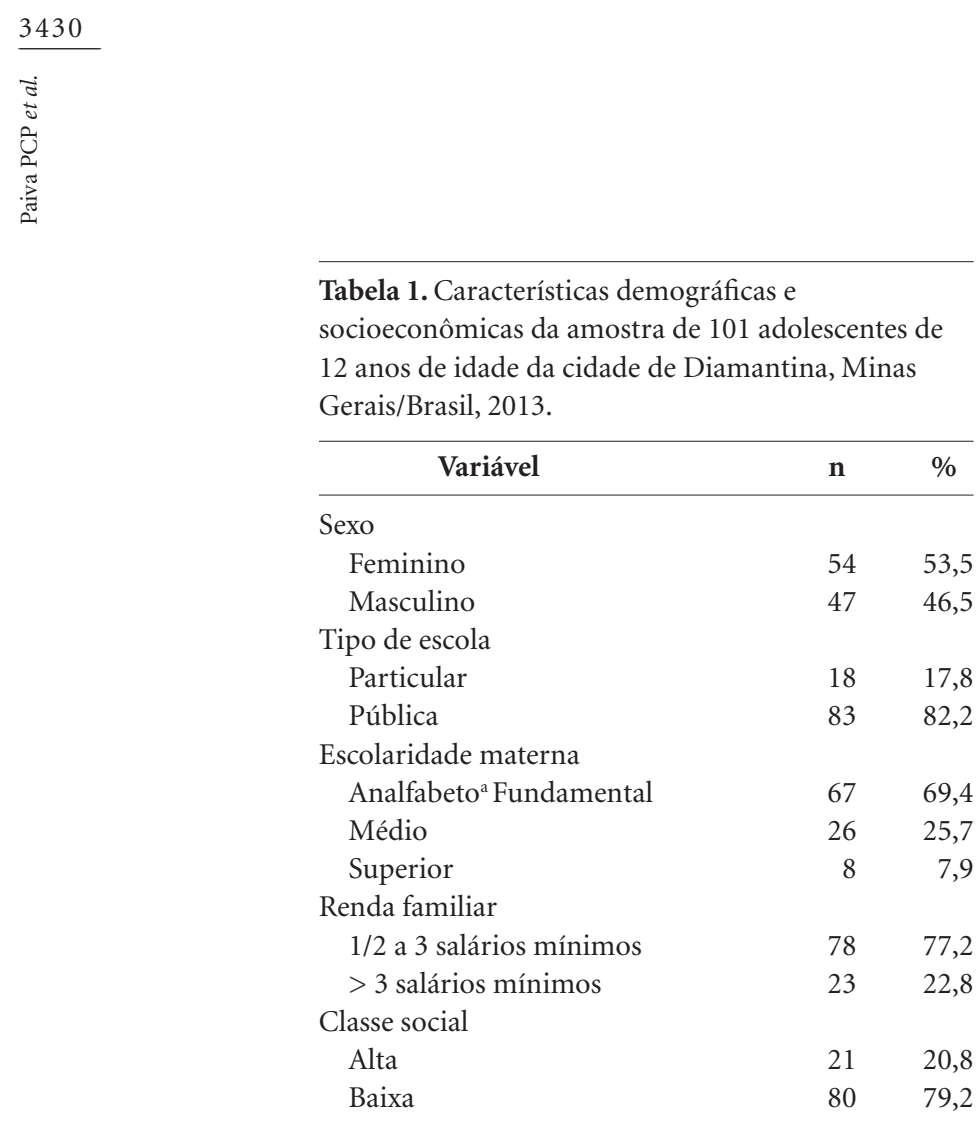

ao grupo de escolares que consumiram bebida alcoólica em "binge".

\section{Discussão}

Para investigar o consumo de bebidas alcoólicas por escolares de 12 anos de idade, foi adotada a versão curta do Instrumento Audit. O Audit-C é um instrumento validado no Brasil ${ }^{20}$, de rápida e fácil aplicação, tendo sido previamente utilizado e considerado adequado para pesquisas envolvendo adolescentes ${ }^{11}$, com a vantagem de considerar os usuários que apresentam problemas iniciais ${ }^{22}$

A prevalência de consumo de bebida alcoólica em "binge" observada no presente estudo foi elevada para a idade dos adolescentes participantes. Prevalência superior foi reportada em outros estudos nacionais ${ }^{11,17,23}$. Entretanto, estas pesquisas foram realizadas em amostra de adolescentes com faixa etária superior a do presente estudo ${ }^{24-26}$. No estudo desenvolvido envolvendo adolescentes da cidade de Belo Horizonte, com idades entre 15 a 19 anos, utilizando metodologia semelhante, a prevalência reportada de consumo abusivo foi de

Tabela 2. Associação entre o consumo abusivo de bebida alcoólica por adolescentes de 12 anos de idade da cidade de Diamantina, Minas Gerais e as variáveis demográficas e socioeconômicas, 2013.

\begin{tabular}{|c|c|c|c|c|c|c|c|}
\hline \multirow{3}{*}{ Variável } & \multicolumn{6}{|c|}{ Consumo abusivo de bebida alcoólica } & \multirow[b]{3}{*}{ Valor $\mathrm{p}$} \\
\hline & \multicolumn{2}{|c|}{ Sim } & \multicolumn{2}{|c|}{ Não } & \multicolumn{2}{|c|}{ Total } & \\
\hline & n & $\%$ & $\mathbf{n}$ & $\%$ & $\mathbf{n}$ & $\%$ & \\
\hline Sexo & & & & & & & $0,274^{*}$ \\
\hline Feminino & 54 & 100 & 43 & 79,6 & 54 & 100 & \\
\hline Masculino & 47 & 100 & 33 & 70,2 & 47 & 100 & \\
\hline Tipo de escola & & & & & & & $0,005^{* *}$ \\
\hline Particular & 18 & 100 & 18 & 100 & 18 & 100 & \\
\hline Pública & 83 & 100 & 58 & 69,9 & 83 & 100 & \\
\hline Renda familiar & & & & & & & $0,054^{* *}$ \\
\hline > 3 salário mínimos & 23 & 100 & 21 & 91,3 & 23 & 100 & \\
\hline 1/2 a 3 salários mínimos & 78 & 100 & 55 & 70,5 & 78 & 100 & \\
\hline Escolaridade materna & & & & & & & $0,024^{* *}$ \\
\hline Superior & 8 & 100 & 8 & 100 & 8 & 100 & \\
\hline Médio & 26 & 100 & 22 & 84,6 & 26 & 100 & \\
\hline Analfabeto a fundamental & 67 & 100 & 47 & 69,2 & 67 & 100 & \\
\hline Controle da mãe & & & & & & & $0,675^{* *}$ \\
\hline Não & 8 & 100 & 7 & 87,5 & 8 & 100 & \\
\hline Sim & 93 & 100 & 69 & 74,2 & 93 & 100 & \\
\hline Controle do pai & & & & & & & $0,098^{*}$ \\
\hline Não & 23 & 100 & 15 & 60,9 & 23 & 100 & \\
\hline Sim & 77 & 100 & 61 & 79,2 & 77 & 100 & \\
\hline
\end{tabular}

* Teste Qui-quadrado de Pearson; ${ }^{* *}$ Teste Exato de Fisher. 
Tabela 3. Associação entre o consumo abusivo de bebida alcoólica por adolescentes de 12 anos de idade da cidade de Diamantina, Minas Gerais e as variáveis demográficas e socioeconômicas, 2013.

\begin{tabular}{|c|c|c|c|c|c|c|c|}
\hline \multirow{3}{*}{ Variável } & \multicolumn{6}{|c|}{ Consumo abusivo de bebida alcoólica } & \multirow[b]{3}{*}{ Valor $\mathrm{p}$} \\
\hline & \multicolumn{2}{|c|}{ Sim } & \multicolumn{2}{|c|}{ Não } & \multicolumn{2}{|c|}{ Total } & \\
\hline & $\mathbf{n}$ & $\%$ & $\mathbf{n}$ & $\%$ & $\mathbf{n}$ & $\%$ & \\
\hline Consumo bebida alcoólica pela mãe & & & & & & & $0,292^{* *}$ \\
\hline Não & 4 & 84,6 & 22 & 15,4 & 26 & 100 & \\
\hline Sim & 21 & 28,0 & 54 & 72,0 & 75 & 100 & \\
\hline Consumo bebida alcoólica pelo pai & & & & & & & $0,264^{* *}$ \\
\hline Não & 3 & 14,3 & 19 & 85,7 & 21 & 100 & \\
\hline Sim & 22 & 28,2 & 57 & 71,8 & 78 & 100 & \\
\hline Consumo bebida alcoólica pelo irmão & & & & & & & $0,056^{*}$ \\
\hline Não & 10 & 17,5 & 47 & 82,5 & 57 & 100 & \\
\hline Sim & 15 & 34,1 & 29 & 65,9 & 44 & 100 & \\
\hline Consumo bebida alcoólica pelo melhor amigo & & & & & & & $0,0001^{* *}$ \\
\hline Não & 1 & 2,6 & 38 & 97,4 & 39 & 100 & \\
\hline Sim & 24 & 38,7 & 38 & 61,3 & 62 & 100 & \\
\hline
\end{tabular}

*Teste Qui-quadrado de Pearson; ${ }^{* *}$ Teste Exato de Fisher.

$36 \%{ }^{11}$. Também foi maior a prevalência de consumo de álcool em estudos internacionais como entre os adolescentes da Nova Zelância ${ }^{9}$, do $\operatorname{Irãa}^{27} \mathrm{e}$ da Argentina ${ }^{28}$. Prevalência semelhante à encontrada na presente investigação foi reportada em estudo com amostra representativa da população estudada, envolvendo 60.973 estudantes do Ensino Fundamental de escolas públicas e privadas das capitais dos estados brasileiros e do Distrito Federal $^{29}$, bem como o estudo realizado na cidade de Pelotas $/ \mathrm{RS}^{30}$. Prevalência inferior de consumo em "binge" foi reportada em amostra de escolares brasileiros com 14 anos $(21,8 \%)$. Os escolares do sul e sudeste apresentaram maior prevalência de "binge" em relação às outras regiões, o que reflete as diferenças culturais existentes em um país de dimensão continental ${ }^{23}$. Assim, a maior prevalência observada em outros estudos nacionais e internacionais seja talvez justificada pelas idades adotadas, pois o consumo tende a aumentar com a idade.

A literatura aponta o sexo masculino como predisponente para o consumo em "bin-

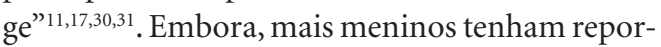
tado consumo em "binge", não houve no presente estudo associação estatísticamente significativa entre sexo e uso de álcool. Esse achado é semelhante ao $1^{\circ}$ Levantamento Nacional Sobre os Padrões de Álcool na População Brasileira, estudo de base populacional com indivíduos entre $14 \mathrm{e}$ 17 anos que também não observou relação esta- tísticamente significante entre os sexos ${ }^{32}$. Talvez uma possível explicação para a falta de associação do consumo em "binge" entre os sexos esteja no comportamento que as meninas vêm adotando na atualidade, bem semelhante aos dos meninos, principalmente na adolescência ${ }^{12}$. O sexo feminino busca melhor engajamento nos grupos de iguais, adotando os mesmos comportamentos na tentativa de maior aceitação ${ }^{12,33}$. Outro fator que deve ser salientado está nas diferenças de desenvolvimento que ocorrem entre os sexos e que pode ser compreendido como algo associado à adolescência em que a maturidade mais rápida entre as meninas reflete sua emancipação e independência ${ }^{23}$ em relação aos meninos, principalmente na idade abordada.

$\mathrm{O}$ ambiente familiar exerce grande influência no início e na manutenção do consumo de drogas lícitas e ilícitas entre adolescentes. Relacionamento ruim com os pais, ser membro de família que usa substância pscicoativa, com pouca comunicação ou que falta suporte e monitoramento familiar têm sido associados ao uso de álcool, tabaco e outras drogas, tornando-se fator de risco para iniciação e/ou manutenção do consumo de substâncias $^{34}$. O consumo em "binge" pelos adolescentes não esteve estatísticamente associado ao consumo de bebida pelos parentes e ao controle dos pais, mas esteve sim ao consumo do melhor amigo. O consumo de bebida alcoólica pelo irmão não foi estatísticamente associado ao beber 
em "binge" pelo adolescente $(0,056)$, porém esteve muito próximo da significância estatística, sugerindo que em amostras maiores esta associação possa ser observada. Fatores sociais, demográficos e ambientais podem aumentar o risco de "beber em binge". O consumo em "binge" pelos adolescentes do presente estudo esteve associado ao consumo de bebidas alcoólicas pelos seus melhores amigos. De acordo com estudo realizado por Gallimberti et al..$^{35}$, um maior risco de consumir bebidas alcoólicas em "binge" por adolescentes esteve associado àqueles que pertencem a um grupo com pouco respeito às regras, ou naqueles nos quais os jovens não são vistos como líderes e que os pares também beberam em "binge", destacando a pressão da socialização exercida pelos pares durante a adolescência. Redes de relacionamento podem influenciar direta ou indiretamente no uso de substâncias entre adolescentes. O conhecimento sobre os relacionamentos é essencial para a compreensão das influências sociais sobre o uso de substâncias, pois os pares tendem a se agrupar com base nas atividades compartilhadas. Assim, o uso de substâncias apareceria como uma relação causal, baseado na influência dos pares sobre o comportamento. Por outro lado, adolescentes podem também selecionar pares com base em padrões semelhantes de comportamento ${ }^{36,37}$.

Para prevenir a exposição dos jovens a esses fatores de risco, destaca-se a importância da família, pois os pais têm um papel importante tanto no controle, quanto no fornecimento de barreiras contras situações potencialmente nocivas $^{35}$.

Resultado semelhante também foi reportado em estudo realizado no estado de São Paulo, onde falta de regras claras sobre o que se pode ou não fazer $(\mathrm{p}=0,034)$ e passar a maior parte do tempo livre com os amigos foram predisponentes para o consumo de bebidas alcoólicas por adolescentes $(p=0,024)$. A adolescência engloba um período crítico no desenvolvimento das relações sociais, no qual os vínculos deixam de ser centrados na família, deslocando-se para a relação com os pares, em que o jovem se sente apoiado pelos colegas, amigos (as) ou namorados (as), compartilhando experiências, emoções e conhecimentos $^{13}$. A experimentação de bebida alcoólica é um importante indicador de monitoramento. Adolescentes cujos pais estão mais atentos às atividades desenvolvidas pelos filhos apresentam menor envolvimento com álcool, drogas e tabaco. A percepção da preocupação dos pais com as atitudes dos filhos desencoraja comportamentos considerados de risco ${ }^{23,38}$.
Não existe consenso na literatura entre o consumo de bebidas alcoólicas e a condição socioeconômica. Alguns estudos encontraram maior consumo de álcool entre os adolescentes pertencentes à classe socioeconômica alta ${ }^{11,17}$, porém, outros reportaram associação entre o consumo de álcool e a classe socioeconômica menos favorecida $^{12-14,18}$, existindo ainda estudos que não encontraram associação estatísticamente significativa $^{30}$. Associação estatística foi observada entre o consumo de bebida alcoólica e os indicadores socieconômicos, tipo de escola, renda familiar e escolaridade materna, corroborando com estudos que reportaram maior consumo entre os adolescentes de classe social menos favorecida. Bellis et al. ${ }^{13}$ reportaram que a facilidade de acesso, a propaganda e o baixo custo das bebidas alcoólicas sejam facilitadores para o aumento do consumo entre os adolescentes e destacam a importância de políticas de monitoramento, principalmente no controle da propaganda e do preço das bebidas.

A idade do primeiro consumo citado pela maioria dos adolescentes $(66,7 \%)$ foi aos 11 anos, o que corrobora com a literatura que vem salientando a precocidade com que os adolescentes estão iniciando o uso de bebidas alcoólicas ${ }^{1}$, mesmo estas sendo proibidas para menores de 18 anos $^{8}$. O início precoce do consumo de álcool desencadeia comportamentos de risco à saúde, tais como o comprometimento do desenvolvimento psicossomático, pobre desempenho escolar, precocidade na iniciação sexual, atos de violência, tentativa de suicídio, uso de drogas ilícitas e dependência na fase adulta ${ }^{12,14,39}$. Dados do CEBRID (Centro Brasileiro de Informações sobre Drogas Psicotrópicas $)^{40}$, com amostra de 17.371 escolares do ensino médio, foram utilizados para testar a hipótese de que o uso precoce de álcool está associado a padrões de consumo abusivo de bebida alcoólica durante a adolescência. Os resultados apontaram que adolescentes que consumiram bebida alcoólica durante a primeira infância foram mais propensos a se envolverem em comportamentos de consumo em "binge" na adolescência, bem como a usarem drogas ilícitas. Baseado nos dados observados, o controle dos pais sobre o uso de álcool pela criança deve ser claro e efetivo, para que ocorra o atraso na idade de experimentação de bebidas alcoólicas ${ }^{17}$, além do desenvolvimento de ações específicas, envolvendo a escola com intuito de prevenir o consumo de bebidas precoce e em "binge" pelos adolescentes.

Não existe um nível seguro de consumo de álcool para crianças e adolescentes ${ }^{14}$. O consu- 
mo de álcool fora do ambiente familiar torna-se ainda mais perigoso, portanto, estratégias para evitar o "binge" em adolescentes devem abordar também a facilidade de acesso e o baixo custo dos produtos, principalmente fora de ambientes supervisionados ${ }^{13}$.

O presente estudo tem limitações que devem ser consideradas. Embora tenha sido garantida a confidencialidade das informações, alguns dados podem ter sido subestimados, pois o constrangimento ou o receio de responder afirmativamente às perguntas pode ter ocorrido ou superestimado, pela vontade de afirmação. As limitações metodológicas do desenho de estudo transversal não permitem inferir causalidade. Além disso, vale ressaltar também, que por envolver uma amostra de conveniência, com idade precoce, dificulta a comparação entre outros estudos, principalmente pela diversidade das realidades, culturas e contextos. Estudos longitudinais e de intervenção são necessários para compreender o consumo em "binge” de álcool entre os adolescentes com amostras maiores abordando suas causas e consequências.

A relevância deste estudo recai sobre a faixa etária, fase inicial da adolescência, de grande vulnerabilidade às influências do ambiente social, incluindo os adolescentes de mais idade. Qual- quer ação de prevenção ou promoção de saúde estaria agindo no início do problema, com chances de potencialização dos resultados.

\section{Conclusões}

O consumo em "binge" foi alto, de início precoce e esteve associado às condições socioeconômicas e ao consumo de bebida alcoólica pelo melhor amigo. A precocidade com que os adolescentes estão iniciando o uso de bebidas alcoólicas é preocupante, mesmo em presença da proibição por lei do consumo por menores e principalmente por estarem inseridos no sistema educacional, onde ações educativas podem ser incorporadas e avaliadas. Fatores sociais, demográficos e ambientais podem aumentar o risco de "beber em binge". O conhecimento sobre os relacionamentos é essencial para a compreensão das influências sociais sobre o uso de substâncias e sua prevenção.

Os resultados apontam para a importância da detecção precoce de consumo em "binge" por adolescentes, possibilitando o planejamento e o desenvolvimento de ações futuras para a prevenção deste consumo nas escolas públicas e privadas, de acordo com seus respectivos contextos.

\section{Colaboradores}

PCP Paiva realizou a coleta dos dados, análise estatística e redação do artigo. HN Paiva realizou a coleta dos dados e redação do artigo. JA Lamounier, EF Ferreira e CAS César realizaram a revisão crítica e intelectual do conteúdo. PM Zarzar participou da concepção do projeto, análise estatística, interpretação dos dados e na revisão crítica do conteúdo. A versão final foi aprovada por todos os autores. 


\section{Agradecimentos}

Este estudo teve o apoio das agências brasileiras de fomento do Conselho de Desenvolvimento Científico e Tecnológico (CNPq), Coordenação de Aperfeiçoamento de Pessoal de Nível Superior (CAPES) e FAPEMIG. Os financiadores não tiveram nenhuma participação no desenho do estudo, coleta de dados e análise, decisão de publicação ou preparação do manuscrito.

\section{Referências}

1. Pratta EMM, Santos MA. Levantamento dos motivos e dos responsáveis pelo primeiro contato de adolescentes do ensino médio com substâncias psicoativas. SMAD. Rev Eletr Saude Mental Alcool Drogas 2006; 2(2):296314.

2. World Health Organization (WHO). Improving Health trough schools: national and international strategies. 1999. [acessado 2014 mar 15]. Disponível em: http:// www.who.int/maternal_child_adolescent/topics/adolescence/dev/en/

3. Senna SRCM, Dessen MA. Contribuições das Teorias do Desenvolvimento Humano para a Concepção Contemporânea da Adolescência. Psicol: Teoria e Pesquisa 2012; 28(1):101-108.

4. Steinberg L, Monahan KC. Age differences in resistance to peer influence. Dev Psychol 2007; 43(6):1531-1543.

5. Véronneau MH, Trempe SC, Paiva AO. Risk and protection factors in the peer context: how do other children contribute to the psychosocial adjustmentof the adolescent? Cien Saude Colet 2014; 19(3):695-705.

6. Parada M, Corral M, Caamaño-Isorna F, Mota N, Crego A, Rodríguez Holguín S, Cadaveira F. Definición del concepto de consumo intensivo de alcohol adolescente (binge drinking). Adicciones 2011; 23(1):53-63.

7. Umberson D, Montez JK. Social relationships and health: a flashpoint for health policy. J Health Soc Behav 2010; 51(Supl. 1):S54-S66.

8. Brasil. Lei $\mathrm{n}^{\circ}$ 9.294, de 15 de julho de 1996. Dispões sobre as restrições de produtos fumíferos, bebidas alcoólicas, medicamentos, terapias e defensivos agrículas. Diário Oficial da União 1996; 15 jul.

9. Teevale T, Robinson E, Duffy S, Utter J, Nosa V, Clark $\mathrm{T}$, Sheridan J, Ameratunga S. Binge drinking and alcohol-related behaviours amongst Pacific youth: a national survey of secondary school students. $N Z$ Med J 2012; 125(1352):60-70

10. Hahm HC, Kolaczyk E, Jang J, Swenson T, Bhindarwala AM. Binge drinking trajectories from adolescence to young adulthood: the effects of peer social network Subs Use Misuse 2012; 47(6):745-756.

11. Zarzar PM, Jorge KO, Oksanen T, Vale MP, Ferreira EF, Kawachi I. Association between binge drinking, type of friends and gender: A cross-sectional study among Brazilian adolescents. BMC Public Health 2012; 12:257.

12. Miller JW, Naimi TS, Brewer RD, Jones SE. Binge drinking and associated health risk behaviors among high school students. Pediatrics 2007; 119(1):76-85.

13. Bellis MA, Phillips-Howard PA, Hughes K, Hughes S, Cook PA, Morleo M, Hannon K, Smallthwaite L, Jones L. Teenage drinking, alcohol availability and pricing: a cross-sectional study of risk and protective factors for alcohol-related harms in school children. BMC Public Health 2009; 9:380.

14. Stolle M, Sack PM, Thomasius R. Binge drinking in childhood and adolescence: epidemiology, consequences, and interventions. Dtsch Arztebl Int 2009 106(19):323-328.

15. Galduróz JCF, Sanchez Zv, Opaleye ES , Noto AR, Fonseca AM, Gomes PL, Carlini EA. Fatores associados ao uso pesado de álcool entre estudantes das capitais brasileiras. Rev Saude Publica 2010; 44(2):267-273. 
16. World Health Organization (WHO). Atlas on substance use. Resources for the prevention and treatment of substance use disorders. 2010. [acessado $2014 \mathrm{fev} 2$ ]. Disponível em: http://www.who.int/features/factfiles/adolescent_health/facts/en/index6.html.

17. Sanchez ZM, Santos MG, Pereira AP, Nappo SA, Carlini EA , Carlini CM , Martins SS. Childhood alcohol use may predict adolescent binge drinking: a multivariate analysis among adolescents in Brazil. J Pediatr 2013; 163(2):363-368.

18. Sanchez ZM, Martins SS, Opaleye ES, Moura YG, Locatelli DP, Noto AR. Social factors associated to binge drinking: a cross-sectional survey among Brazilian students in private high schools. BMC Public Health 2011; 31(11):201.

19. Mattara FP, Ângelo PM, Faria JB, Campos JADB. Confiabilidade do Teste de Iidentificação de Transtornos Devido ao Uso de Álcool (Audit) em Adolescentes. Rev Electronica Salud Ment Alcool Drogas 2010; 6(2):296314.

20. Meneses-Gaya C, Zuardi AW, Loureiro SR, et al. Is the full version of the Audit really necessary? Study of the validity and internal construct of its abbreviated versions. Alcohol Clin Exp Res 2010; 34(8):1417-1424.

21. Jander A, Mercken L, Crutzen R, Vries Hd. Determinants of binge drinking in a permissive environment: focus group interviews with Dutch adolescents and parents. BMC Public Health 2013; 24(13):882.

22. Reinert DF, Allen JP. The alcohol use disorders identification test: an update of research findings. Alcohol Clin Exp Res 2007; 31(2):185-199.

23. Costa MCO, Alves MVQM, Santos CAST, Carvalho RC, Souza KEP, Sousa HL. Experimentação e uso regular de bebidas alcoólicas, cigarros e outras substâncias psicoativas SPA na adolescência. Cien Saude Colet 2007; 12(5):1143-1154.

24. Sanceverino SL, Abreu JLC. Aspectos epidemiológicos do uso de drogas entre estudantes do ensino médio no Município de Palhoça 2003. Cien Saude Colet 2004; 9(4):1047-1056.

25. Schenker M, Minayo MCS. Fatores de risco e de proteção para o uso de drogas na adolescência. Cien Saude Colet 2005; 10(3):707-717.

26. Baheiraei A, Hamzehgardeshi Z, Mohammadi MR, Nedjat S, Mohammadi E. Alcohol and drug use prevalence and factors associated with the experience of alcohol use in Iranian adolescents. Iran Red Crescent Med J 2013; 15(3):212-217.

27. Pierobon M, Barak M, Hazrrati S, Jacobsen KH. Alcohol consumption and violence among Argentine adolescents. J Pediatr 2013; 89(1):100-107.

28. Malta DC, Mascarenhas MDM, Porto DL, Duarte, Eliane Aparecida; Sardinha LM, Barreto SM, Morais Neto OL. Prevalência do consumo de álcool e drogas entre adolescentes análise dos dados da Pesquisa Nacional de Saúde Escolar. Rev Bras Epidemiol 2011; 14(Supl. 1):136-146.
29. Strauch ES, Pinheiro RT, Silva RA, Horta BL. Uso de álcool por adolescentes: estudo de base populacional. Rev Saude Publica 2009; 43(4):647-655.

30. Malta DC, Machado IE, Porto DL, Silva MM, Freitas PC, Costa AW, Oliveira-Campos M. Alcohol consumption among Brazilian Adolescents according to the National Adolescent School-based Health Survey (PeNSE 2012). Rev Bras Epidemiol 2014; 17(Supl. 1):203-214.

31. Campos JADB, Almeida JdC, Garcia PPNS, Faria JB. Consumo de álcool entre estudantes do ensino médio do município de Passos - MG. Cien Saude Colet 2011; 16(12):4745-4754

32. Pinsky I, Zaleski M, Laranjeira R. Primeiro Levantamento Nacional sobre os Padrões de Consumo de Álcool na População Brasileira. Rev Bras Psiquiatr 2010; 32(3):214-215.

33. Vieira PC, Aerts DRGC, Freddo SL, Bittencourt A, Monteiro L. Uso de álcool, tabaco e outras drogas por adolescentes escolares em município do Sul do Brasil. Cad Saude Publica 2008; 24(11):2487-2498.

34. Malbergier A, Cardoso LRD, Amaral RAd. Uso de substâncias na adolescência e problemas familiares. Cad Saude Publica 2012; 28(4):678-688.

35. Gallimberti L, Chindamo S, Buja A, Buja A, Forza G, Tognazzo F, Galasso L, Vinelli A, Baldo V. Underage drinking on saturday nights, sociodemographic and environmental risk factors: a cross-sectional study. Subs Abuse Treat Prev Policy 2011; 6:15.

36. Fujimoto K, Valente TW. Alcohol peer influence of participating in organized school activities: a network approach. Health Psychol 2013; 32(10):1084-1092.

37. Valente TW, Gallaher P, Mouttapa M. Using Social Networks to Understand and Prevent Substance Use: A Transdisciplinary Perspective. Subs Use Misuse 2004; 39(10-12):1685-1712.

38. Paiva FS, Ronzani TM. Estilos parentais e consumo de drogas entre adolescentes: revisão sistemática. Psicol Estudo 2009; 14(1):177-183.

39. Crabbe JC, Harris RA, Koob GF. Preclinical studies of alcohol binge drinking. Ann N Y Acad Sci 2011; 1216:24-40

40. Centro Brasileiro sobre Drogas Psicotrópicas (CEBRID). Departamento de Psicobiologia - Unifesp/ EPM Bebidas alcóolicas. Unifesp. [acessado 2014 jun 21]. Disponível em: http://www.unifesp.br/dpsicobio/ cebrid/folhetos/alcool .htm.

Artigo apresentado em 01/09/2014

Aprovado em 21/11/2014

Versão final apresentada em 23/11/2014 
